\title{
《Review》 \\ Biological Features of the Avian Male Gamete and their Application to Biotechnology of Conservation
}

\author{
Elisabeth Blesbois \\ INRA, UMR-PRC, 37380 Nouzilly, France
}

\begin{abstract}
Male gamete biology shows specific features in birds that contribute to the reproductive strategy of flying animals adapted to highly variable environmental conditions. In this paper, we review the main characteristics of bird sperm, their selection and storage in the highly specialized female oviduct, specific features of polyspermic fertilization, and the consequences of these biological adaptations on semen biotechnology. Among other features, the storage of sperm in specific oviducal glands is a key factor to increase reproductive "freedom" but is also a critical point for the success of semen in vitro storage.
\end{abstract}

Key words: bird, fertilization, sperm, storage

J. Poult. Sci., 49: 141-149, 2012

\section{Introduction}

Birds have developed original reproductive processes which are very efficient for the adaptive strategies of flying animals to highly variable environmental conditions. Oviparity has evolved to the production of telolecithal, hardshelled eggs, enabling the autonomous development of the embryo in a highly protected micro-environment. The highly specialized egg production results in the formation of a single oviduct in the adult female of most bird species. The oviduct shares two main functions: 1) the storage and selection of sperm prior to the polyspermic fertilization of the oocyte in the infundibulum; and 2) the constitution of the outer vitelline membrane, albumen and shell of the egg that contributes to the autonomy of the embryo development. The oviduct is also the site of zygote formation and divisions up to stage 30-50000 cells when the embryo genome is activated at the time of oviposition. Among other features, the presence of sperm storage tubules (SSTs, see review in Bakst et al., 1994, Blesbois and Brillard, 2007) in the uterovaginal junction (UVJ) dedicated to the prolonged storage of sperm provides "adaptive freedom" to the female to sustain fertilizing potential even in the absence of males. Sperm are produced by the two intra-abdominal testis of the males at body temperature $\left(41-43^{\circ}\right)$, a fact that may be related to the short interval of time required for spermatogenesis (reviewed by Aire, 2007). For example, in the chicken, guinea-fowl, duck and turkey, the duration of spermatogenesis is approx-

Received: October 4, 2011, Accepted: January 10, 2012

Released Online Advance Publication: March 25, 2012

Correspondense: Dr. E. Blesbois, INRA, UMR-PRC, 37380 Nouzilly, France. (E-mail: Elisabeth.Blesbois@tours.inra.fr) imately 14 days which provides males with extended potential to produce large quantities of gametes within a given period of time. Following their evacuation from the rete testis, avian sperm are rapidly transported through the epididymis to the vas deferens, where they are stored for a limited period of time (e.g. 2-3 days in the chicken, Williams and de Reviers, 1981) prior to being exported at ejaculation. Unlike in mammalian species, there is no sexual accessory gland in the vas deferens of birds. The rapidity and low specialization of the transport of bird sperm in the male tract is in contrast with the high complexity of their selection and storage in the female (from 10 days storage in the quail up to $70 \mathrm{~d}$ in the turkey) (reviewed by Aire, 2007). Sperm storage in the female tract has been used to develop original reproductive adaptation. Thus, the choice of sires with high reproductive potential based on the preferential selection and storage of their sperm has probably developed in wild species (Birkhead and Brillard, 2007). In domestic species, the development of genealogic selection in the poultry industry has been followed by the standardization of egg and meat production. However the control of reproductive performances is still not optimal and the production, quality and efficiency of storage of the male gamete have not been widely taken into account. This has now resulted in conventional breeding in early decreases in reproductive performance and in major welfare problems (reviewed by Hammerstedt, 1999; Brillard, 2004; De Jong and Guemene, 2011).

The process of in vivo sperm storage in the oviduct is also a challenge to the development of appropriate methodologies to preserve sperm in vitro, as avian sperm must still undergo the normal processes of selection and prolonged storage in the SSTs following in vitro storage. Procedures to store 
poultry semen in vitro in a liquid state were first developed to facilitate the management of breeder flocks subjected to artificial insemination (AI) and also to transport genetic material to distant locations without the need for setting up of local breeder male facilities. Cryopreservation has been considered to be an alternative strategy to limit the erosion of genetic diversity and is also of major interest for the preservation of endangered breeds as it greatly facilitates the development of international programs to assess genetic biodiversity. Recent advances in cryopreservation technology for poultry semen have resulted in the emergence of cryobanking which is now being developed in an increasing number of countries (reviewed by Blesbois, 2007; Blesbois et al., 2010; Blesbois, 2011).

In the present review we will develop major aspects of specific features of bird sperm biology and their selection for fertilization, and the consequences on reproduction strategies and on reproductive biotechnology.

\section{The Male Gamete at Ejaculation}

Throughout the reproductive cycle, the key aim of male gamete production in birds is to produce "the highest number of gametes for the lowest reproductive cost". Following the rapid spermatogenesis, spermatozoa remain for a short time (1-3 days) in the male tract without annex gland. The signs of sperm maturation are much less developed than in mammals. Unlike mammals, chicken spermatozoa already possess most of their motility capacity and a significant part of their fertilizing capacity when they leave the testis (Howarth, 1983; Ashizawa and Sano, 1990). These capacities are then increased in the epididymis before transit in the deferent duct. At the time of ejaculation the number of spermatozoa per ejaculate is usually very high $\left(0.5^{-1}\right.$ billion in the chicken; 2 billion in the turkey) but concentrated in small volumes (less than $0.5 \mathrm{ml}$ ). In many species, the phallus is "vestigial" or has totally disappeared as in all the galliform order.

Bird spermatozoa show characteristics that are common to all the amniote species and also specific adaptations to their complex system of internal fertilization. These thin gametes (Fig. 1) possess a head, an intermediate piece and a long flagellum (reviewed by Aire, 2007). The head contains a small acrosome $(2.5 \mu \mathrm{m}$ long and $0.5 \mu \mathrm{m}$ wide in the chicken $)$ and the nucleus $(6 \times 0.5 \mu \mathrm{m}$ in the chicken). The acrosome includes the acrosome vesicle that contains different hydrolytic enzymes such as acrosin, involved in the digestion of the inner perivitelline layer (IPVL) that surrounds the oocyte and the perforatorim, a rigid actin-based structure with an unknown role in birds. The nucleus, surrounded by its double nuclear membrane, is extremely condensed, a protection to the genome during the very long passage in the oviduct before the fusion with the oocyte. In the Passeriformes, a helicoidal sheath surrounds the head. The intermediate piece contains a proximal and a distal centriole and also a highly variable number of mitochondria (approximately 30 mitochondria in the chicken, 150 in the Japanese quail) that may extend some distance into the flagellum depending on the species. It is the "motor" of the energetic metabolism of these gametes that do not contain significant intracellular reserves but must be very motile to access the female SST and then the oocyte and must also stay alive for a number of weeks in the female tract (Fig. 2). The flagellum is the longest part of the cell (70-90 $\mu \mathrm{m}$ in the chicken). It starts at the level of the distal centriole and contains an axoneme with one central doublet and nine peripheral microtubule doublets, mainly constituted of tubulin. In most birds (except Passeriformes), the axoneme is surrounded by a fibrous sheath in most of the flagellum. At ejaculation spermatozoa are diluted in seminal plasma that comes from diffuse secretions and excretions of the male tract (reviewed by Etches, 1996). This biological medium presents a neutral (or slightly basic) $\mathrm{pH}$ and its osmolarity is sustained by a high content of amino acids such as glutamate and different proteins, the most abundant being a serum-like albumin (Blesbois and Caffin, 1992). It contains high levels of cholesterol and phospholipids originating from numerous lipidic and lipoproteic (high density lipoproteins, very high density lipoproteins) vesicules (Blesbois and Hermier, 1990; Douard et al., 2000). Seminal plasma stimulates sperm motility and is useful at the time of ejaculation. It is not a good medium of sperm storage despite the presence high molecular weight fractions that sustain fertility, and is eliminated before the sperm reach the SST (Blesbois and de Reviers, 1992).

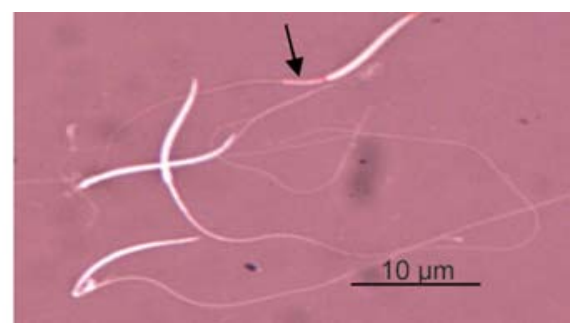

Fig. 1. Chicken sperm observed with optical microscope. Chicken sperm observed under optical microscope on an eosin-nigrosin smear (photos I. Grasseau, INRA, France): left=one viable and morphologically normal sperm; 2 a right $=$ abnormal sperm (coiled or bent flagella, one sperm with intermediate piece permeable to eosin with arrow). 


\section{2a Hen oviduct:}

\section{2b}

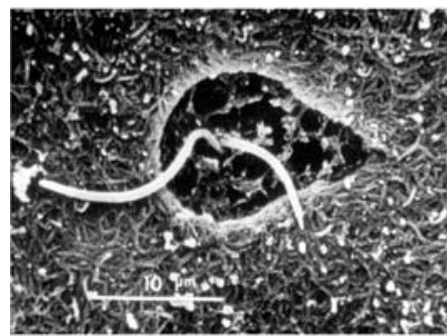

2 sperm through the IPVL (Bakst, 1994)

\section{Hen oviduct:}

\section{In Vivo Storage and Selection of Sperm in the Female Tract}

At mating, sperm are either deposited into the cloaca ("cloacal kiss") in species such as chickens and turkeys, or introduced into the mid-vagina in species with a retractable penis (ostrich, duck, goose). Sperm are then subjected to a drastic process of vaginal selection prior to being stored in the SSTs. Only $1 \%$ of the initial sperm population reaches the utero-vaginal junction (UVJ) (Brillard and Bakst, 1990). As only $1 \%$ of the sub-population of pre-selected sperm can be further recovered in the inner perivitelline layer (IPVL), it can be postulated that the overall population of pre-selected sperm transported to the site of fertilization does not exceed $1 / 1,000^{\text {th }}$ of the initial population deposited in the vagina (reviewed by Blesbois and Brillard, 2007). Thus the female oviduct would act as a succession of biological filters hind- ering the migration and storage of low quality or damaged sperm. The first filter is the access to the UVJ-SSTs. Despite the existence of a secondary site of storage of spermatozoa in the infundibulum, it is generally recognized that UVJ-SSTs are the main sperm storage site in poultry as most sperm stored for prolonged periods of time are located at this site. These sperm storage tubules are cylindrical, generally non-branched structures found in the folds of the upper vaginal mucosa. Their numbers are species-dependent, ranging from less than 200-500 in passerines (Briskie and Montgomerie, 1993) to 20,000 in the turkey (Goodrich-Smith, 1977) and may be genetically selected to increase the storage length (Brillard et al., 1998). It has long been accepted that sperm that reach the SSTs must be motile (reviewed by Bakst et al., 1994). Sperm with high motility show higher rates of success after heterospermic insemination than sperm with low motility, and their advantage increases with the time of 
storage in the SSTs (Pizzari et al., 2008). Motility has also been suggested to continue during the stay in the SSTs where sperm have to maintain position against a fluid current generated by the SST epithelial cells, and motility would also be involved in their release (Froman, 2003). Immunological status of sperm and oviduct are also important for sperm selection. Steele and Wishart (1992) showed that immunoglobulin identification of sperm could be involved in the access to SSTs. More recently, a series of studies suggested a potential role of cytokines in the elimination of "undesirable" sperm in the vagina and the immune protection of transforming growth factor- $\beta$ s in the SSTs (Das et al., 2006; 2009).

\section{Contribution of Sperm to the Fertilization Process}

The initial event of fertilization in birds is the fixation of numerous sperm on the IPVL that surrounds the oocyte at ovulation. The fixation is followed by the acrosome reaction (AR), an exocytosis process involving the fusion between the sperm plasma membrane and the outer acrosomal membrane (Okamura and Nishiyama, 1978a). N-Glycanes fractions of the IPVL ZP proteins and specific arrangement of ZP3 fractions would be involved in the sperm fixation (Horrock et al., 2000; Han et al., 2010; Wassarman, 2010). But the specificity is low and other perivitelline layer fractions i.e. the outer perivitelline are also efficient (Lemoine et al., 2008). The fixation of sperm to the perivitelline layer induces the activation of different signaling pathways (sperm PI3-kinase followed by PKA and MAPK1) and leads to acrosome reaction (Lemoine et al., 2009). The AR leads to the liberation of proteolytic acrosomic enzymes such as acrosin that help the digestion of the IPVL and also the mobilization of intra acrosomal calcium that could be involved in different processes including the oocyte activation. Calcium plays a central role in the AR. Extra-cellular calcium is an absolute requirement for $\mathrm{AR}$ and is found in abundance in the oviduct. Specific "Calcium storage structures" have also been described in the infundibulum of chicken and quail (Rabbani et al., 2006; 2007). Calcium is also involved as second messenger in the successive steps of the AR in all animal species with internal fertilization and its liberation from the intra-acrosomic stones would also be involved (Roldan, 2007).

Many sperm may undergo AR and then penetrate the bird's oocyte after the fusion between the inner membrane of the acrosome and the plasma membrane of the oocyte (Okamura and Nishiyama, 1978b). They preferentially penetrate near to but not on the germinal disc and then evolve to the formation of male pronuclei (reviewed by Stepinska and Bakst, 2007). The pronuclei formation involves profound changes in the sperm nuclei, including chromatin decondensation, disappearance and then neo formation of the nuclear membrane. However, only one male pronucleus is involved in the final syngamy. The role of polyspermy in birds is still in question. One hypothesis involves the amount of phospholipase $\mathrm{C}$ zeta (PLC $\zeta$ ) produced by sperm that is needed to activate the oocyte. Indeed, the presence of PLC $\zeta$ in chicken sperm has been proven (Coward et al., 2005). The injection of chicken PLC $\zeta$ RNA into mice oocytes induced egg activation (Dong et al., 2000). The injection of quail sperm and PLC $\zeta$ RNA or of higher concentration of PLC $\zeta$ RNA alone has proven its efficiency to induce the formation of the quail female pronucleus (Mizushima et al., 2008; 2009). The existence of a positive dose effect of PLC $\zeta$ RNA on the rate of early embryo development lead to suggest that polyspermy could help to increase the rate of PLC $\zeta$ liberated in the oocyte and needed for an optimal activation of the egg. This could be an adaptive strategy related to the very large size of the telolecithal oocyte. A higher chance of activation of the oocyte would be given by polyspermy.

\section{Biotechnology of Conservation}

The general and the specific features of the biology of sperm in birds have been taken as a basis to develop biotechnologies that may be used to extend the period of time between the production of the male gametes and their use in the formation of zygotes. Most of these technologies derive from the use of ejaculated sperm. One exception is the transfer and storage of spermatogonial cells (Trefil et al., 2006; 2010).

Extending the time between production and utilization of the male gamete permit two main types of action:

1) Manage the diffusion of the initial genetic potential of the males;

2) Include an in vitro incubation that may be used profitably to modify the property of sperm, including changes in their genetic potential.

Until very recently the first method met with some success in birds but not the second. Although very recent results (Mizushima et al., 2010) suggest that ICSI of sperm treated with triton X-100 could be successful in the quail, suggesting future applications for gene transfer, this approach is as yet barely used. The very efficient DNAse of bird oocytes has been suggested to rapidly eliminate "foreign" materials and is suspected to be involved in the poor success of these methods (Stepinska and Bakst, 2007). Thus we will now focus this chapter on the more widely used methods of semen storage. Semen in vitro storage must be done very carefully in birds because every small defect in the in vitro procedures will be exacerbated during the long process of semen in vivo storage in the female oviduct. The sperm that have been previously stored in vitro make up part of the populations that may be rapidly eliminated in the oviduct. Four main factors are important to ensure the success of in vitro storage. (a) The in vitro media must share physical properties compatible with cell life. (b) As bird sperm are cells without intra-cellular reserves, the sperm energetic metabolism is one of the main sources of problems during and after in vitro storage. The course of intracellular metabolism also includes the management of free radicals and peroxides. (c) The plasticity of the sperm membrane to resist osmotic and temperature changes induced by in vitro storage is an absolute requirement to retain the biological properties of sperm (d) The last crucial question is the maintenance of the capacity of the 
Table 1. Specific parameters of freezing semen handling and insemination depending on species (Seigneurin and Blesbois, 2010).

\begin{tabular}{lccccc}
\hline \hline & Chicken & Muscovy Duck & Pekin Duck & Goose & Guinea Fowl \\
\hline $\begin{array}{l}\text { Dilution rate } \\
(\% \text { semen })\end{array}$ & $25-40$ & 65 & 35 & 70 & 35 \\
\hline $\begin{array}{l}\text { AI Dose } \\
\left(\times 10^{6} \text { sperm }\right)\end{array}$ & 400 & 300 & 300 & 40 & 200 \\
\hline $\begin{array}{l}\text { AI Frequency } \\
(/ \text { week })\end{array}$ & 2 & 2 & 2 & 3 & 2 \\
\hline
\end{tabular}

sperm to undergo the acrosome reaction because birds are species in which the AR may be induced very rapidly in vitro and does not need highly specific media. This means that AR would be inhibited by in vitro storage media.

These factors are important for liquid semen storage at temperatures above $0^{\circ} \mathrm{C}$ and also for cryopreservation. However, the dependence on energetic metabolism will be greater with liquid storage and the resistance of the plasma membrane to osmotic stress will be the main problem with cryopreservation (reviewed by Blesbois and Brillard, 2007).

(a) Among other characteristics, semen diluents must provide relatively constant physico-chemical stability to sperm during in vitro storage. Iso-osmotic and nearly neutral $\mathrm{pH}$ conditions must be ensured. This is generally achieved by mixing balanced salt solutions in the osmolarity range $320-$ 450 milliosmoles and the $6.8-7.4 \mathrm{pH}$ range. Decreases in $\mathrm{pH}$ during storage may indicate an increase in sperm glycolysis by products such as lactic acid, while an increase in $\mathrm{pH}$ has been reported to be related to increasing numbers of dead sperm.

(b) At the time of ejaculation, avian sperm contain very few intra-cellular energy reserves, but repeated studies have demonstrated that they can access various types of metabolite "fuels" present in the extra-cellular medium to ensure their basal metabolism and motility. The energy metabolites available to sperm vary between species, but they generally consist mainly of sugars (mainly fructose or glucose) and fatty acids found in seminal plasma and the oviduct, including SSTs. As a consequence, energy substrates may be added to diluents in order to prolong sperm viability and activity in vitro. At the same time, sperm membranes must be protected against free radicals and peroxides. The addition of antioxidants such as vitamin $\mathrm{E}$ and selenium to semen diluents has been found effective to counteract the action of free radicals (Blesbois et al., 1993; Dimitrov et al., 2006). Peroxidation occurs more rapidly under quite high storage temperatures $\left(15\right.$ to $41^{\circ} \mathrm{C}$, Donoghue and Donoghue, 1997; Douard et al., 2004), and when the males are aging (Douard et al., 2003). However, the fertilizing potential of sperm kept in vitro at body-like temperatures $\left(41^{\circ} \mathrm{C}\right.$ for chicken) cannot be maintained over many minutes. In order to improve in vitro storage conditions, sperm metabolism must be decreased. This is generally achieved by reducing the temperature. (c) The general principle guiding the choice of in vitro storage temperature conditions for avian semen is "the longer the duration of storage, the lower the temperature". However the sperm plasma membranes are very rich polyunsaturated fatty acids (PUFAs) fractions that present certain specificity in birds. They contain mainly n-6 PUFAs and always contain n-9 PUFAs even after dietary changes in chickens and turkeys (Blesbois et al., 1997; Douard et al., 2000; 2003; 2004). They are highly susceptible to lipid peroxidation that may be limited by anti-oxidant supplementation (see b). They are also rapidly submitted to local phase transitions during storage at "infra-physiological" temperatures that may alter their membrane fluidity and plasticity. Membrane fluidity and resistance to osmotic stress induced by temperature changes are species-specific (Blanco et al., 2000; Blesbois et al., 2005). Membrane fluidity also depends on the sperm phospholipid composition and on the phospholipids/cholesterol ratios. They may be modified to a certain extent by in vivo (diet, place in the reproduction cycle) or in vitro (cholesterol or phospholipid supply or depletion) conditions (reviewed by Blesbois and Hermier, 2003) and are affected by freezing (Fig. 3). Membrane fluidity of fresh semen added to motility and viability may predict $85 \%$ of the variability of fertility obtained with frozen-thawed semen (Table 2). In addition, a progressive decrease in temperature (e.g. $0.5^{\circ} \mathrm{C} / \mathrm{min}$ ) helps to prevent adverse cold shock effects. However, even under optimal conditions of liquid storage, significant losses of the main plasma membrane phospholipids are observed in the chicken and the turkey (Blesbois et al., 1999; Douard et al., 2000). The endogenous phospholipases have been suspected to play a part in the degradation process (Douard et al., 2004).

Sperm plasma membranes are submitted to specific osmotic stress during cryopreservation. During the freezing steps, the cells are subjected to successive reductions in volume due to dehydration and internal cryoprotectant action. Then during the thawing process, swelling of the sperm with rehydration and expulsion of the cryoprotectant from the intra-cellular fraction, and also a transient increase in intra-cellular ice particle size induces very high tensions in all cell membranes. The nuclear membrane seems quite well resistant, but mitochondrial and plasma membranes are highly sensitive to these physical stress. The membrane injuries that occur during the freeze-thaw process lead to the death of 


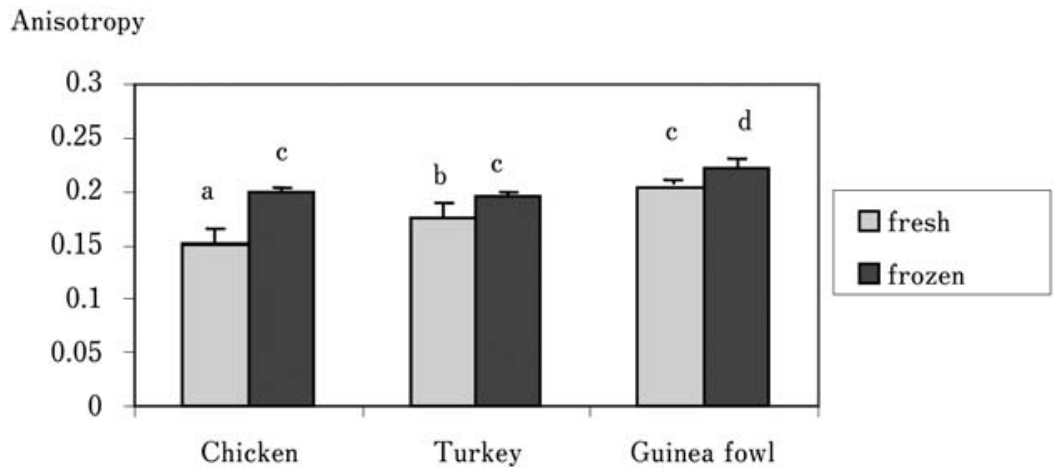

Fig. 3. Membrane fluidity, lipids, ability to cryopreservation (Blesbois et al., 2005). Anisotropy in the opposite of fluidity: the higher the anisotropy (reprnsent rigidity) and the lower the fluidity. Thus sperm fluidity is higher in chicken than in turkey and guinea fowl and the highest rigidity is found in guinea fowl $(p \leq 0.05)$. Freeze-thaw process increases significantly the sperm rigidity.

Table 2. Correlation between quality of fresh semen and fertility obtained with frozen-thawed semen (Blesbois $e t$ al., 2008).

\begin{tabular}{lc}
\hline \hline & Correlation coefficient (Pearson) \\
\hline A: Membrane Fluidity & $0.80(P \leq 0.001)$ \\
\hline B: Viable-normal sperm (\%) & $0.76(P \leq 0.001)$ \\
\hline C: Motile sperm (\%) & $0.64(P \leq 0.01)$ \\
\hline Subjective motility & $0.61(P \leq 0.02)$ \\
\hline $\begin{array}{l}\text { Fertility with fresh sperm (fixed } \\
\text { number of sperm inseminated) }\end{array}$ & $0.74(P \leq 0.01)$ \\
\hline
\end{tabular}

Correlation between quality of fresh semen and fertility obtained with frozen-thawed sperm $\mathrm{r} 2 \mathrm{~A}+\mathrm{B}+\mathrm{C}=0.85$

more than $50 \%$ of sperm, to membrane rigidifying, metabolic defects with mobility alteration and ATP decrease and changes in the plasma membrane glycocalyx (Long, 2006; Blesbois et al., 2008; Peleaz and Long, 2008). Most of these changes do not seem to show localization in their action on the plasma membrane, but the acrosomal cap could show specific reactivity.

(d) Acrosome reaction shares specific conditions in birds when compared to mammals. Specific features lead to huge differences in the sensitivity of bird spermatozoa to in vitro storage. In most mammalian species, acrosome reaction is preceded by sperm capacitation with major changes in the plasma membrane (calcium entry, cholesterol removal, changes in phospholipids and glycocalyx) that prepare sperm for AR. In vitro storage show "capacitation-like "effects that may be very useful for in vitro fertilization in mammals. In the absence of capacitation in birds, in vitro storage alters the capacity of chicken sperm to undergo the acrosome reaction (Lemoine et al., 2011). During liquid storage, the alteration of AR capacity is more rapid and much stronger that the alteration of the other sperm functions such as viability and motility. We may thus now suggest that the capacity to undergo the AR is a prerequisite for the success of liquid storage of bird sperm.

The acrosome reaction is also frequently altered by the freezing process in birds. But conversely to liquid storage, AR capacity seems to be only secondarily affected by cryopreservation. Indeed, in high quality semen, AR is much less altered than motility and viability (Lemoine et al., 2011). Finally, when present, the alteration of AR is irreversibly induced by the first contact with the internal cryoprotectant (CPAs) (Mocé et al., 2010). We may thus suggest that the interaction with the CPAs is critical for maintaining the capacity of sperm to undergo AR after cryopreservation. Consequently, AR induction would be a key test when deciding how to add CPAs prior to the freezing process.

All these elements show that bird AR shares very specific process that may help to emphasize the differences occurring in sperm quality changes during liquid and freezing semen storage. However the nature of the changes is different in liquid storage and in cryopreservation and these two storage methods have very different applications: liquid storage is extensively used in the practice of artificial insemination while semen freezing is used in the conservation of genetic diversity.

\section{Liquid Storage and Artificial Insemination}

Artificial insemination (AI) technology needs preservation of sperm viability and fertilizing potential under in vitro conditions over short or extended periods of time. AI practice in the poultry industry expanded for selection and multiplication purposes over the second half of the twentieth century but its justification for the production of broiler-type chicks remains species-specific. For example, the turkey industry switched from natural mating to $\mathrm{AI}$ in the 1960s because of the strong negative phenotypic correlation between breast widths and mating potential (reviewed by Krueger, 2003). AI has also become increasingly popular to produce guinea fowl chicks because the hens are not suited to trap nesting 
Table 3. Examples of fertility obtained with cryopreserved semen from chicken (Blesbois et al., 2007).

\begin{tabular}{lcc}
\hline \hline \multicolumn{1}{c}{ Genetic origin } & Homologous females & Commercial females \\
\hline Gauloise dorée breed & $21 \%^{\mathrm{b}}$ & $39 \%^{\mathrm{a}}$ \\
\hline $\begin{array}{l}\mathrm{R}+\text { experimental line } \\
\text { (high heat production line) }\end{array}$ & $14 \%^{\mathrm{c}}$ & $25 \%^{\mathrm{b}}$ \\
\hline $\begin{array}{l}\mathrm{B} 4 \text { experimental line (subfertile } \\
\text { line with specific histocompati- } \\
\text { bility haplotype) }\end{array}$ & $7 \%^{\mathrm{d}}$ & $43 \%^{\mathrm{a}}$ \\
\hline a, b, c, d represent significant differences $(P \leq 0.05)$. &
\end{tabular}

conditions. AI has also become extensively used to produce mule ducklings (male Muscovy X female Pekin), a consequence of the repeated problems of mating behavior encountered in heterospecific breeder flocks. AI is now widespread in the poultry industry for selection purposes, but it has also become increasingly popular to produce broiler chicks in regions with low labor costs. In all these cases, semen is recommended to be stored for very few hours to avoid a decrease in fertility (6-24 h in the chicken). However, as previously described, the length of storage will depend on the storage conditions and on the physiological state of the animals (Blesbois and Brillard, 2007).

\section{Semen Freezing and Cryobanking}

The extensive use of semen freezing for current reproduction practice remains unrealistic, partly due to the high cost of employing frozen compared to fresh semen and partly due to the difficulty of obtaining freeze/thaw avian semen without significant loss of fertilizing potential. As previously described, in the case of sperm treated for cryopreservation, damage caused to plasma membranes results in a dramatic increase in sperm rejection prior to storage in the SSTs, thus preventing subsequent fertilization (reviewed by Blesbois and Brillard, 2007). Such impairment of fertility parameters may be partly compensated by increasing the number of sperm deposited at each insemination and also by increasing the frequency of insemination (Table 1; Seigneurin and Blesbois, 2010). However, even under optimal conditions, high intra-line and inter-line (Table 3 ) variability does exist, mainly related to the initial semen quality and female fertility (Blesbois et al., 2007; Blesbois et al., 2008). Surprisingly, embryo mortality following insemination with frozenthawed spermatozoa is generally below that observed with fresh semen, at least in the chicken (Seigneurin and Blesbois, 1995). This indicates that freeze/thaw procedures may 'emphasize' the natural process of sperm selection exerted by the lower oviduct prior to their acceptance in the SSTs.

With species-specific zootechnical adaptations, semen freezing has successfully been used in chickens, geese, and pekin and muscovy ducks (reviewed by Blesbois, 2011). Taking as models the methods developed in domestic birds (diluent, cryoprotectant, freezing and thawing rate), sperm freezing is also now under development in a small number of wild species including wild pheasianides, Barbary partridge and Griffon vultures, although fertility results are still lacking (Saint Jalme et al., 2003; Madeddu et al., 2009; Madeddu et al., 2010). The successes obtained in domestic species open up the possibility of creating national programs that include the storage of genetic diversity in the form of sperm. The main advantage of the use of semen for this purpose is the non-invasive procedure developed to collect, treat and transfer the cells to the female through AI. Thus, despite the fact that semen will still share only half of the total genome, it still remains the main cell type to be used for breed reconstruction, creation of synthetic breeds, gene introgression and use for live-cryo-aided schemes. In most national cryobanking programs, sperm cells are completed by the additions in the cryobanks of alternative diploid cells with reproductive potential such as Blastodermal and primordial or gonadic germ cells (reviewed by Blesbois, 2011).

However the progress made in the last 15 years in this area concerns a small number of domestic or wild bird species. This still has to be developed in others such as turkeys, guinea fowl and quails.

\section{Conclusion}

Bird sperm show original adaptations to their complex system of internal fertilization. The rapid and rather unspecific fabrication of mature sperm in the adult male contrasts with the highly specific process to which sperm are submitted in the oviduct, including highly efficient selection and long storage in specific glands. The polyspermic fertilization of the telolecithal egg in birds also shares specific features that will affect the induction of the acrosome reaction and penetration of the ovum, the selection of the male pronucleus to be involved in the syngamy and the action of egg DNAses.

These specific features contribute to the "adaptive freedom" of bird reproductive systems. They have also been successfully used to optimize the management of males in the poultry industry and to develop methods of conservation of genetic resources in national programs of cryobanking.

New basic knowledge of birds' semen biology are however needed to understand what are the main factors involved in the selection of the sperm to be used for fertilization and consequently lead to zygote formation. Application of sperm biology to methods of semen storage should also be extended 
to a larger number of species and to new approaches for gene transfer.

\section{References}

Aire TA. Spermatogenesis and testicular Cycles. In Reproduction Biology and Phylogeny of Birds. (Jamieson BGM ed.), Queensland Univ. Queensland, Australia. pp. 279-349. 2007.

Ashizawa K and Sano R. Effects of temperature on the immobilization and the initiation of motility of spermatozoa in the reproductive tract of the domestic fowl. Comparative Biochemistry and Physiology 96: 297-301. 1990.

Bakst MP. Fate of fluorescent stained sperm following insemination. Biology of Reproduction, 50: 987-992. 1994.

Birkhead TR and Brillard JP. Reproductive isolation in birds: postcopulatory prezygotic barriers. Trends in Ecology and Evolution, 22: 266-272. 2007.

Blanco JM, Gee G, Wildt DE and Donogue AM. Species variation in osmotic, cryoprotectant, and cooling rate tolerance in poultry, eagle, and falcon spermatozoa. Biology of Reproduction, 63: 1164-1171. 2000.

Blesbois E and Hermier D. Effects of high-density lipoproteins on storage at 4 degrees $\mathrm{C}$ of fowl spermatozoa. Journal of Reproduction and Fertility, 90: 473-482. 1990.

Blesbois E and Caffin J.P. "Serum like" albumin of fowl seminal plasma and effects of albumin on fowl spermatozoa stored at 4 degrees C. British Poultry Science, 33: 663-670. 1992.

Blesbois E and de Reviers M. Effect of different fractions of seminal plasma on the fertilizing ability of fowl spermatozoa stored in vitro. Journal of Reproduction and Fertility, 95: 263-268. 1992.

Blesbois E, Grasseau I and Blum JC. Effects of vitamin E on fowl semen storage at 4 degrees C. Theriogenology, 39: 771-779. 1993.

Blesbois E, Lessire M, Grasseau I, Hallouis JM and Hermier, D. Effect of dietary fat on thefatty acid composition and on fertilizing ability of fowl semen. Biology of Reproduction, 56: 1216-1220. 1997.

Blesbois E, Grasseau I and Hermier D. Changes in lipid content of fowl spermatozoa after liquid storage at 2 to 5 degrees C. Theriogenology, 52: 325-334. 1999.

Blesbois E and Hermier D. Specificity of fatty acids in domestic birds spermatozoa. In: Phospholipids and Male Fertility. (De Vriese S ed.). Chap. 7. pp. 73-85. Champaign. 2003.

Blesbois E, Grasseau I. and Seigneurin F. Membrane fluidity and the ability to survive cryopreservation in domestic bird spermatozoa. Reproduction, 129: 371-378. 2005.

Blesbois E. Current status in avian semen cryopreservation. World's Poultry Science Journal, 63: 213-222. 2007.

Blesbois E and Brillard JP. Specific features of in vivo and in vitro sperm storage in birds. Animal, 1: 1472-1481. 2007.

Blesbois E, Seigneurin F, Grasseau I, Limouzin C, Besnard J, Gourichon D, Coquerelle G and Tixier-Boichard M. Semen cryopreservation for ex-situ management of genetic diversity in chicken. Poultry Science, 87: 555-564. 2007.

Blesbois E, Grasseau I, Seigneurin F, Mignon-Grasteau S, Saint Jalme M and Mialon-Richard MM. Predictors of semen cryopreservation in chickens. Theriogenology 69: 252-261. 2008.

Blesbois E, Seigneurin F, Tixier-Boichard M and Pain B. Strategy of avian germplasm cryobanking in France. Proc. 13 European Poultry Conference (EPC 2010). World's Poultry Science Journal. 2010, 66 (S numéro spécial: Supplément); p: 246. CD rom, 5 pages. 2010 .
Blesbois E. Freezing avian semen. Avian Biology Review, 4: 44-50. 2011.

Brillard JP and Bakst MR. Quantification of spermatozoa in the sperm-storage tubules of turkey hens and its relation to sperm numbers in the perivitelline layer of eggs. Biology of Reproduction, 43: 271-275. 1990.

Brillard JP, Beaumont C and Scheller MF. Physiological responses of hens divergently selected on the number of chicks obtained from a single insemination. Journal of Reproduction and Fertility, 114: 111-117. 1998.

Brillard JP. Natural mating in broiler breeders: present and future concerns. World's Poultry Science Journal, 60: 439-445. 2004.

Briskie JV and Montgomerie R. Patterns of sperm storage in relation to sperm competition in passerine birds. Condor, 95: 442-454. 1993.

Coward K, Ponting CP, Chang HY, Hibbitt O, Savolainen P, Jones $\mathrm{KT}$ and Parrington J. Phospholipase $\mathrm{C} \zeta$, the trigger of egg activation in mammals, is present in non-mammalian species. Reproduction, 130: 157-163. 2005.

De Jong IC and Guemene D. Major welfare issues in broiler breeders. World's Poultry Science Journal, 67: 73-81. 2011.

Das SC, Isobe N, Nishibori M and Yoshimura Y. Expression of transforming growth factor $\beta$-isoforms and their receptors in utero-vaginal junction of hen oviduct in presence or absence of resident sperm with reference to sperm storage. Reproduction, 132: 781-790. 2006.

Das SC, Isobe $\mathrm{N}$ and Yoshimura $\mathrm{Y}$. Changes in the expression of interleukin- $1 \beta$ and lipopolysaccharide-induced TNF factor in the oviduct of laying hens in response to artificial insemination. Reproduction, 137: 527-536. 2009.

Dimitrov SG, Atanasov VK, Surai PF and Denev SA. Effect of organic selenium on Turkey semen quality during liquid storage. Animal Reproduction Science, 100: 311-317. 2006.

Dong JB, Tang TS and Sun FZ. Xenopus and chicken sperm contain a cytosolic soluble protein factor which can trigger calcium oscillations in mouse eggs. Biochemica Biophysica Research Communication, 268: 947-951. 2000.

Donoghue A and Donoghue D. Effects of water and lipid-soluble antioxidants on turkey sperm viability, membrane integrity and motility during liquid storage. Poultry Science, 76: 14401445. 1997.

Douard V, Hermier D and Blesbois E. Changes in turkey semen lipids during liquid in vitro storage. Biology of Reproduction, 63: $1450-1456.2000$.

Douard V, Hermier D, Magistrini M, and Blesbois E. Reproductive period affects lipid composition and quality of fresh and stored spermatozoa in turkeys. Theriogenology, 59: 753-764. 2003.

Douard V, Hermier, D, Magistrini M, Labbé C and Blesbois E. Impact of composition changes in the storage medium on lipids and quality of turkey spermatozoa. Theriogenology, 61: 1-13. 2004.

Etches RJ. The male. In Reproduction in Poultry. Eds Wallingford, London, UK pp 208-233. 1996.

Froman D. Deduction of a Model for Sperm Storage in the Oviduct of the Domestic Fowl (Gallus domesticus). Biology of Reproduction, 69: 248-253. 2003.

Goodrich-Smith M and Marquez BJ. A technique for studying the utero-vaginal sperm storage tubules. Poultry Science, 56: 1718. 1977.

Hammerstedt RH. Symposium summary and challenges for future. Poultry Science, 78: 459-466. 1999. 
Han L, Monné M, Okumura H, Schwend T, Cherry AL, Flot D, Matsuda T and Jovine L. Cell, 143: 404-415. 2010.

Horrocks AJ, Stewart S, Jackson L and Wishart GJ. Induction of acrosomal exocytosis in chicken spermatozoa by inner perivitelline-derived N-linked glycans. Biochemistry and Biophysics Research Communications, 278: 84-89. 2000.

Howarth JR. Fertilizing ability of cock spermatozoa from the testis, epididymis and vas deferens following intramagnal insemination. Biology of Reproduction, 28: 586-590. 1983.

Krueger K. Tom mangement-past, present and future. In: Fifth International Symposium on Turkey Reproduction. Raleigh (USA), 2003/10/1-3. pp 75-83. 2003.

Lemoine M, Grasseau I, Brillard JP and Blesbois, E. A reappraisal of factors involved in the in vitro induction of acrosome reaction in chickens. Reproduction, 136: 391-399. 2008.

Lemoine M, Dupont J, Guillory V, Tesseraud S and Blesbois E. Signaling pathways involved in the chicken acrosome reaction. Biology of Reproduction, 81: 657-665. 2009.

Lemoine M, Mignon-Grasteau S, Grasseau I, Magistrini M and Blesbois E. Capacity of chicken spermatozoa to undergo the acrosome reaction after in vitro storage. Theriogenology, 75: 122-130. 2011.

Long JA. Avian semen cryopreservation: what are the biological challenges? Poultry Science, 85: 232-236. 2006.

Madeddu M, Berlinguer F, Ledda M, Leoni GG, Satta V, Succu S, Rotta A, Pascui V, Zinellu A, Muzzeddu M, Carru C and Naitana S. Ejaculate collection efficiency and post-thaw semen quality in wild-caught Griffon vultures from the Sardinian population. Reproductive Biology and Endocrinology. 7, 18. 2009.

Madeddu M, Berlinguer F, Ledda M, Pascui V, Succu S, Satta V, Leoni GG, Zinellu A, Muzzeddu M, Carru C and Naitana S. Differences in semen freezability and intracellular ATP content between the rooster and the Barbary partridge. Theriogenology, 74: 1010-1018. 2010.

Mizushima S, Takagi S, Ono T, Atsumi Y, Tsukada A, Saito N and Shimada K. Developmental enhancement of intracytoplasmic sperm injection (ICSI)-generated quail embryos by phospholipase C $\zeta$ cRNA. Journal of Poultry Science, 45: 152-158. 2008.

Mizushima S, Takagi S, Ono T, Atsumi Y, Tsukada A, Saito N and Shimada K. Phospholipase C $\zeta$ mPRNA expression and its potency during spermatogenesis for activation of quail oocyte as a sperm factor. Molecular Reproduction and Development, 76: 1200-1207. 2009.

Mizushima S, Takagi S, Ono T, Atsumi Y, Tsukada A, Saito N, Sasanami T, Okabe M and Shimada K. Novel method of Gene transfer in Birds: intracytoplasmic sperm injection for green fluorescent protein expression in quail blastoderms. Biology of Reproduction, 83: 965-969. 2010.

Mocé E, Grasseau I and Blesbois E. Cryoprotectant and FreezingProcess alter the Ability of Chicken Sperm to Acrosome React. Animal Reproduction Science, 122: 359-366. 2010.

Okamura F and Nishiyama $\mathrm{H}$. The passage of spermatozoa through the vitelline membrane in the domestic fowl, Gallus gallus. Cell Tissue Res, 188: 497-508. 1978a.

Okamura F and Nishiyama H. Penetration of spermatozoa into the ovum and transformation of the spermatozoon nucleus into the male pronucleus in the domestic fowl. Cell and Tissue Research, 190: 89-98. 1978b.

Pelaez J and Long JA. Characterizing the Glycocalyx of poultry semen: II In vitro storage of turkey semen and mobility phenotype affects the carbohydrate component of sperm membrane glycoconjugates. Journal of Andrology, 29: 431-439. 2008.

Pizarri T, Worley K, Burke T and Froman D. Sperm competition dynamics: ejaculate fertilizing efficiency changes differentially with time. BMC Evolutionary Biology, 8: 332. 1-7. 2008.

Rabbani MG, Sasanami T, Mori M and Yoshizaki N. Sperm-egg interaction is mediated by a sperm-associated body in quail. Development, Growth \& Differentiation, 48: 33-40. 2006.

Rabbani MG, Sasanami T, Mori M and Yoshizaki N. Characterization of the sperm-associated body and its role in the fertilization of the chicken Gallus domesticus. Development, Growth \& Differentiation, 49: 39-48. 2007.

Roldan. Sperm phospholipases and acrosomal exocytosis. Frontiers in Biosciences, 1: 89-104. 2007.

Saint Jalme M, Lecoq R, Seigneurin F, Blesbois E and Plouzeau, E. Cryopreservation of semen from endangered pheasants: the first step towards cryobank for endangered avian species. Theriogenology, 59: 875-888. 2003.

Seigneurin $\mathrm{F}$ and Blesbois E. Effects of the freezing rate on viability and fertility of frozen-thawed fowl spermatozoa. Theriogenology, 43: 1351-1358. 1995.

Seigneurin F and Blesbois E. Proc. 13 European Poultry Conference (EPC 2010). World's Poultry Science Journal. 2010, 66 (S numéro spécial: Supplément); p: 172.CD Rom 5p. 2010.

Steele MG and Wishart GJ. Evidence for a species-specific barrier to sperm transport within the vagina of chicken hens. Theriogenology, 38: 1107-1114. 1992.

Stepinska U and Bakst MR. Fertilization. In: Reproductive Biology and Phylogeny of Birds. (Jamieson BGM ed.) vol. 10. pp. 553-587. 2007.

Trefil P, Mičáková A, Mucksov, J, Hejnar J, Bakst MR, Poplštein M, Kalina, J. and Brillard JP. Restoration of spermatogenesis and male fertility by transplantation of dispersed testicular cells in the chicken. Biology of Reproduction, 75: 575-581 2006.

Trefil P, Bakst MR, Yan H, Hejnar J, Kalina J and Mucksova J. Restoration of spermatogenesis after transplantation of $\mathrm{c}-\mathrm{Kit}$ positive testicular cells in the fowl. Theriogenology, 74: 16701676. 2010.

Wassarman PM and Litscher ES. Egg's ZP3 structure speaks volumes. Cell, 143: 337-338. 2010.

Williams $J$ and de Reviers M. Variations in the plasma levels of luteinizing hormone and androstenedione and their relationship with the adult daily sperm output in cockerels raised under different photoschedules. Reproduction Nutrition Development, 21: 1125-1135. 1981. 